\title{
AMPA-induced Excitotoxic Lesions of the Basal Forebrain: A Significant Role for the Cortical Cholinergic System in Attentional Function
}

\author{
J. L. Muir, ${ }^{1}$ B. J. Everitt, ${ }^{2}$ and T. W. Robbins ${ }^{1}$ \\ Departments of 'Experimental Psychology and ${ }^{2}$ Anatomy, Universily of Cambridge, Cambridge, CB2 3EB, United \\ Kingdom
}

The aim of the present study was to clarify the role of the basal forebrain (BF)-cortical cholinergic system in visual attentional function by investigating the effect of $\alpha$-amino3-hydroxy-5-methyl-4-isoxazole propionic acid (AMPA)-induced lesions of the basal forebrain on performance of a five-choice serial reaction time task. AMPA lesions in the present study produced a profound effect on performance of the task, as measured by choice accuracy and correct response latency. This deficit was significantly greater than that observed in earlier studies following ibotenate- or quisqualate-induced lesions of the BF. However, detailed histological and biochemical analysis revealed three rather different BF lesions depending upon the batch of AMPA supplied. In one group of animals (BF/1) the deficits in task performance were substantially greater and longer lasting compared to another group of lesioned animals (BF/2), which showed behavioral recovery several months following the lesion. The former sustained severe pallidal damage in addition to marked reductions in cortical ChAT activity. Support for the attentional nature of these deficits was obtained by the ability to improve task performance in BF/1 lesioned animals by increasing the duration of the visual stimulus and thus reducing the attentional load placed on these animals. In contrast, performance deficits could be reinstated in those animals showing behavioral recovery $(B F / 2)$ by reducing the duration of the visual stimulus and thus increasing attentional load.

In the second experiment more discrete lesions of the magnocellular cholinergic neurons were made, resulting in extensive reduction of cortical ChAT activity with considerably less neuronal loss from the dorsal pallidum compared to the BF/1 lesion group. Once again, deficits on the task were substantially greater than observed previously following either quisqualate- or ibotenate-induced BF lesions. Furthermore, the cholinergic specificity of these deficits was supported by the attenuation of behavioral impairments following administration of the anti-cholinesterase physostigmine.

Taken together with our earlier work, which has failed to demonstrate mnemonic deficits following lesions to the mag-

\footnotetext{
Recerved June 15, 1993: revised Sept. 20, 1993; accepted Oct. 4, 1993

This work was supported by a Ploglanne Giant from the Wellcome Trust

Correspondence should be addressed to Janice L. Muir, Department of Experimental Psychology, University of Cambridge. Downing Street, Cambridge, CB2 $3 \mathrm{~EB}, \mathrm{UK}$.

Copyright 1994 Society for Neuroscience $0270-6474 / 94 / 142313-14 \$ 05.000$
}

nocellular neurons of the nucleus basalis of Meynert, these results suggest that the most consistent deficit produced following lesions of the BF-cortical cholinergic system is attentional dysfunction. Analogous deficits in visual attention are also seen in patients with Alzheimer's disease, which can also be improved by anti-cholinesterase treatment.

[Key words: ACh, attention, basal forebrain, AMPA, Alzheimer's disease]

Degeneration of the cholinergic magnocellular neurons of the nucleus basalis of Meynert (nbM) and their cortical projections is a major feature of the neuropathology of Alzheimer's disease as well as Parkinson's disease and progressive supranuclear palsy (Agid et al., 1987). The decline in memory as well as other cognitive deficits associated with Alzheimer's dementia has been attributed, at lcast in part, to degeneration of this $n b M-n e o-$ cortical cholinergic system (Bartus et al., 1982: Coyle et al., 1983). Therefore, it is not surprising that the contribution of the cortical cholinergic system to learning and memory function has recently been the subject of considerable investigation and controversy (for reviews, see Dunnett et al. 1991; Fibiger, 1991). Indeed, the behavioral cffects of excitotoxic lesions of the basal forebrain (BF), targeted on the $\mathrm{nbM}$, have been investigated on a wide range of behavioral tasks and the impairments observed have generally been interpreted as supporting a role for this system in learning and memory.

The lack of a selective cholinergic neurotoxin has meant that the majority of these studies have used axon-sparing excitotoxic amino acids, in particular ibotenic acid, to destroy BF cholinergic neurons. However, once a more effective cholinergic neurotoxin, such as quisqualic acid, was employed, many of the learning and memory impairments observed following ibotenic acid-induced lesions of the BF were no longer apparent. This was despite the fact that quisqualate-induced lesions produced greater destruction of cortically projecting cholinergic neurons of the basal forebrain and significantly greater reductions in cortical ChAT activity than ibotenic acid (Dumnell el al., 1987; Etherington et al., 1987; Robbins et al., 1989a,b).

Furthermore, histological analysis has revealed that excitotoxic doses of NMDA receptor agonists, such as ibotenic acid, damage many noncholinergic neurons within the BF, especially dorsal and ventral pallidal neurons (Page et al., 1991). In contrast, quisqualic acid appears to damage the other basal forebrain neurons less readily while destroying cholinergic neurons within the same area (Dunnett et al., 1987; Robbins et al., $1989 \mathrm{a}, \mathrm{b})$. On the basis of such evidence, Dunnett et al. (1991) have recently proposed that many of the memory and learning 
impairments observed following ibotenic acid lesions of the BF are not attributable to destruction of cholinergic neurons of the nucleus basalis, but rather may occur as a result of disruption to corticostriatal outputs coursing through the dorsal and ventral globus pallidus.

Indeed, it appears that many aspects of cognitive function remain unaffected following disruption of the cortical cholinergic system with relatively more specific lesions of the BF (Dunnett et al., 1987; Wenk et al., 1989). However, using a fivechoice serial reaction time task, deficits in visual attentional function have consistently been observed following quisqualic acid lesions of the BF (Robbins et al., 1989b; Muir et al., 1992a). Using this paradigm, Robbins et al. (1989b) reported that rats with basal forebrain lesions were impaired in their ability to localize brief visual targets, as measured by choice accuracy and latency to respond correctly to the visual stimulus. However, in this study, quantitatively as well as qualitatively similar behavioral impairments were observed in the performance of ibotenate- and quisqualate-lesioned animals. On the basis of these data alone it is therefore difficult to conclude that the cortical cholinergic system is essential for attentional function, given the disparity between the degree of reduction in cortical ChAT activity and the equivalent behavioral effects obtained using these two excitotoxins.

One approach taken in attempting to resolve this issue has been to transplant cholinergic-rich embryonic cells into the deafferented cortex of animals with lesions of the basal forebrain. Amelioration of quisqualate-induced behavioral deficits, in particular the disruptive effects of white noise on task performance, has been observed using this technique (Muir et al., 1992a). A second approach has shown similar behavioral deficits to those observed with BF lesions following intrabasalis infusions of the GABA agonist muscimol (Muir et al., 1992b). Importantly, the muscimol-induced impairments could be reversed by systemic administration of the anti-cholinesterase physostigmine.

Most recently, in attempting a more selective means of destroying the basal forebrain cholinergic neurons, $\alpha$-amino-3hydroxy-5-methyl-4-isoxazole propionic acid (AMPA), the prototypical agonist at the AMPA subtype of non-NMDA glutamate receptor, has been characterized (see Page et al., 1991). This excitatory amino acid has been shown to be even more effective than quisqualic acid in destroying cholinergic magnocellular neurons, producing a $70 \%$ reduction in cortical ChAT activity, while sparing dorsal pallidal neurons and also many noncholinergic neurons located within the ventral pallidum (Page et al., 1991). Despite this large reduction in cortical ChAT activity, Page et al. report that both acquisition and performance of spatial learning in the Morris water maze were unaffected by the AMPA lesion, in contrast to the significant impairments observed on this task following ibotenic acid lesions of the basal forebrain.

The aim of the present study was thcreforc to clarify the role of the cortical cholinergic system in attentional function by investigating the effect of $A M P A$, this seemingly potent and more selective neurotoxin for cholinergic neurons, on attentional performance using the five-choice task, which we have shown previously to be susceptible to disruption by both ibotenate- and quisqualate-induced lesions of the basal forebrain (Robbins et al., 1989b; Muir et al., 1992a,b). Furthermore, in order to infer cholinergic specificity of the AMPA-induced impairments on this task, reversal of the behavioral deficits was attempted by administration of the anti-cholinesterase physostigmine.

\section{Materials and Methods}

\section{Animals}

Male Lister hooded rats (Olac, Bicester, UK) were housed in pairs in a temperature-controlled $\left(21^{\circ} \mathrm{C}\right)$ room, under natural daylight conditions, with water available ad libitum. Rats were food deprived and maintained at $90 \%$ of their free-feeding weight (MRC Diet $41 \mathrm{~B}$ laboratory chow) throughout the experiment.

\section{Surgical procedures}

Animals were anesthetized using Equithesin administered intraperitoneally $(0.3 \mathrm{ml} / 100 \mathrm{gm})$ and placed in a Kopf stereotaxic instrument fitted with atraumatic earbars. In experiment 1,15 rats received bilateral injections of 0.015 м AMPA ( $\alpha$-amino-3-hydroxy-5-methyl-4-isoxazole propionic acid; Cambridge Rescarch Biochemicals, UK; batches 06175 and 02774 , proportion of + and - enantiomers unknown) and seven sham-operated controls received infusions of sterile $0.2 \mathrm{~m}$ phosphate buffer ( $\mathrm{pH}$ 7.2). A volume of $0.5 \mu \mathrm{l}$ of AMPA or the same volume of buffer alone was infused bilaterally over $90 \mathrm{sec}$ via a 30 gauge cannula attached to a $5 \mu$ l precision sampling syringe (SGE, Baton Rouge, LA) at each of two placements: (1) AP $+0.2 \mathrm{~mm}$ from bregma, $\mathrm{L} \pm 3.4 \mathrm{~mm}$ from the midline, and DV $-7.0 \mathrm{~mm}$ below the dura; and (2) AP +1.0 $\mathrm{mm}$ from bregma, $\mathrm{L} \pm 2.6 \mathrm{~mm}$ from the midline, and $\mathrm{DV}-7.3 \mathrm{~mm}$ (incisor bar set at $5.0 \mathrm{~mm}$ above the interaural line). The injection cannula was left in place for a further $2 \mathrm{~min}$ following each infusion. Shortly after the completion of surgery, those rats receiving AMPA lesions exhibited minor seizures and were treated with diazepam $(3 \mathrm{mg}$ / kg, i.p.) where necessary.

However, following detailed histological analysis of the lesion after completion of this initial study, it became apparent that there was substantial variability in the potency of AMPA that was attributable to variability of different batches supplied of this compound. In particular, the high degree of nonspecific pallidal damage caused by the excitotoxin in this first experiment was batch dependent. This had not been observed previously either in pilot studies or in an earlier study by Page et al. (1991), where a different batch of the compound had been employed. Consequently, in experiment 2 , an attempt was made to produce a more selective basal forebrain cholinergic lesion, similar to the one characterized by Page et al. (1991), in order to investigate the cholinergic specificity of the behavioral impairments obtained by administration of the acetylcholinesterase inhibitor physostigmine. By reducing the concentration of AMPA for this particular batch (batch 08403) to 0.0075 $M$, a pilot study revealed widespread loss of cholinergic neurons of the Ch4 cell group while sparing nonmagnocellular neurons within the pallidum. Accordingly, six rats received lesions of the basal forebrain using this particular concentration of AMPA, administered in the sante volume and at the same sites as described for experiment 1. A second group of six animals served as sham controls and received phosphate buffer alone.

\section{Choline acetyltransferase (ChAT) activity in the cerebral cortex}

At the conclusion of behavioral testing, animals were decapitated and the brains rapidly removed and placed onto an ice-cooled plate. Samples of medial frontal cortex, anterior dorsolateral frontal neocortex, cingulate and parietal cortices, as well as hippocampus were dissected and stored at $-70^{\circ} \mathrm{C}$ for subsequent assay of ChAT activity using the method of Fonnum (1975).

Medial frontal cortex was taken as the area extending $3 \mathrm{~mm}$ caudal to the frontal polc and $1 \mathrm{~mm}$ laterally from the midline, the caudal surface of this area therefore coinciding with $+2.0 \mathrm{~mm}$ from bregma. This medial frontal cortex sample comprised areas $\mathrm{Cg} 1, \mathrm{Cg} 3$, and $\mathrm{Fr} 2$ according to the classification of Zilles (1985). The anterior dorsolateral cortex was taken as the lateral area of cortex from this initial slice, extending laterally to the rhinal fissure, and included Zilles' areas Fr1, Fr2, Fr3, and Par1.

The first sample of postgenu cingulate cortex (cingulate 1) included the medial cortex extending 3-5 $\mathrm{mm}$ from the frontal pole (caudal surface coinciding with bregma) and $1 \mathrm{~mm}$ laterally from the midline. This sample contained areas $\mathrm{Cg} 1, \mathrm{Cg} 2$, and $\mathrm{Fr} 2$. Posterior dorsolateral frontal cortex was taken from this lateral extent to the point immediately above the rhinal fissure and comprised of areas Fr1, Fr3, FL, and Par1. The second sample of postgenu cingulate cortex (cingulate 2) encompassed the medial cortex from bregma and extended $2 \mathrm{~mm}$ posterior to bregma. This area therefore included $\mathrm{Cg} 1, \mathrm{Cg} 2, \mathrm{Fr} 2$, and a small section 
of RSG. Parietal cortex (Fr1, HL, FL, Parl, and Par2) was taken from the lateral extent of this cingulate sample to the point immediately above the rhinal fissure. Finally, a section of hippocampus was carefully dissected.

\section{Histology}

Following removal of cortex for the measurement of ChAT activity, brains were fixed by immersion in $10 \%$ formol saline. Following fixation and storage in $30 \%$ sucrose, the brains were sectioned on a freezing microtome at $60 \mu \mathrm{m}$ thickness. Every fourth section through the region of the lesion was mounted on a glass slide for staining with cresyl violet. These sections were used to verify lesion placement and to assess the extent of lesion-induced neuronal loss.

\section{General behavioral procedures}

Apparatus. The test apparatus for these experiments consisted of three $25 \times 25 \mathrm{~cm}$ aluminum chambers built in the Department of Experimental Psychology, University of Cambridge. The rear wall of each chamber was concavely curved and contained nine apertures, each 2.5 $\mathrm{cm}$ square, $4 \mathrm{~cm}$ deep, and set $2 \mathrm{~cm}$ above floor level. Illumination of each hole was provided by a standard $3 \mathrm{~W}$ bulb located at the rear of the hole. In addition, each hole had an infrared photocell beam monitoring the entrance and each hole could be blocked by a metal cover when not required (for details, see Carli et al., 1983).

The three chambers were individually housed within wooden soundattenuating cabinets, ventilated by low-level noise fans, which also served to mask extraneous background noise. Each chamber was illuminated by a $3 \mathrm{~W}$ house light mounted in the center of the roof alongside a small general-purpose loud speaker. White noise could be delivered through the speaker by a purpose-built white noise generator.

Animals were placed in the chamber through a Perspex door located in the front wall. Directly below this door, animals obtained access to the food magazine by pushing a hinged Perspex panel monitored by a microswitch. Food pellets ( $45 \mathrm{mg}$, dustless; Bioserv, Inc., NJ) were dispensed automatically into the magazine. The distance from the magazine panel to each of the holes in the rear wall was $25 \mathrm{~cm}$.

The apparatus and on-line data collection were controlled by means of a Control Universal (Cambridge, UK) Cube microcomputer system with software written in ONLIBASIC.

Behavioral procedure. Rats were trained to discriminate a brief visual stimulus presented randomly in one of five spatial locations, as described previously (Robbins ct al., 1989b). The training procedure began with two 15 min sessions with the response apertures covered with metal caps. During these sessions, the magazine panel was partially open and food pellets were placed in the tray. In the next two $30 \mathrm{~min}$ sessions, the metal caps were removed from five of the apertures (from left: 1 , $3,5,7,9)$ and several food pellets placed within each aperture as well as within the food tray. During the fifth session the test schedule was implemented.

At the beginning of each test session, the house light was illuminated and free delivery of a single food pellet to the magazine was made. The trial was initiated by the rat opening the panel to collect this pellet. After a fixed $5 \mathrm{sec}$ intertrial interval (ITI), the light at the rear of one of the apertures was illuminated for a short period $(0.5 \mathrm{sec})$. Responses in this aperture during illumination and for $5 \mathrm{sec}$ afterward (the limited hold period) were rewarded with the delivery of a food pellet and a correct response was recorded. Additional responses in the apertures were recorded as perseverative responses and resulted in a $5 \mathrm{sec}$ period of darkuless (time-out). Further responding in the apertures during the time-out restarted this period. Responses in a nonilluminated hole during the signal period (incorrect response) and failures to respond within the limited hold period (omission) were similarly punished with a period of darkness. Once again, responses made in an aperture during this period restarted the time-out.

A response in the food panel after the delivery of a food pellet, or after the time-out period, initiated the next trial. Additional responses in the panel during the ITI or time-out periods were recorded but had no further consequences. Responses in the apertures during the ITI were recorded as anticipatory responses and resulted in a time-out period of darkness, additional responses during this time restarting the time-out period.

During any one session, the light stimulus was presented an equal number of times in each of the five holes in a randum order. A daily session consisted of 100 trials or was terminated after $30 \mathrm{~min}$ of testing.
The end of a test session was signaled by extinguishing all the lights. For the first session of training, the stimulus duration and limited hold periods were both set at $1 \mathrm{~min}$, and the ITI and time-out periods set at $3 \mathrm{sec}$. These variables were altered on subsequent trials according to the individual animal's performance, until the target set of task parameters could be instituted. The target parameters were as follows: stimulus duration, $0.5 \mathrm{sec}$; limited hold period, $5 \mathrm{sec}$; ITI and time-out period, $5 \mathrm{sec}$. The animals were considered to have reached criterion when these target parameters were attained on five consecutive sessions with $>80 \%$ correct responses and $<20 \%$ omissions within the $30 \mathrm{~min}$ session time Approximately 30 sessions were required for the animals to attain this criterion.

\section{Performance assessment}

Performance of the task was assessed using the following behavioral measures.

Accuracy. The accuracy of performance was measured as the proportion of responses that were correct (number of correct responses/ total number of responses), expressed as a percentage.

Speed. Two measures of speed of responding were used. The first was the latency to respond correctly, defined as the time between the onset of the visual stumulus and the point at which the animal's nose breaks the infrared beam of the lit hole. The second measure was magazine latency: the time between performance of a correct response and the opening of the magazine panel to collect the food pellet.

Anticipatory responses. The number of responses in the apertures during the I'TI.

Perseverative responses. Additional responses in the apertures following the initial response in an aperture.

Errors of omission. The number of trials on which no response was made during the limited hold period.

\section{Statistical methods}

Data for each variable were subjected to analysis of variance (ANOVA) using the GENSTAT (Rothamsted, UK) statistical package. Further post hoc comparisons were made using the Newman-Keuls test. Skewed data, which violate the distribution requirement of the ANOVA, were subjected to arcsine, square root, or logarithmic transformation as recommended by Winer (1971).

\section{Experiment 1: Effects of AMPA Lesions of the Basal Forebrain on Attentional Function}

\section{Behavioral procedures}

Three weeks following surgery, the 22 animals used in this experiment were tested across five sessions on the standard schedule of the task. Animals were once again tested on this schedule 14 weeks following lesion surgery in order to assess the persistence of the behavioral impairments, as previous research (Muir et al., 1992a) has revealed behavioral recovery of task performance at this time point following quisqualic acid lesions of the $\mathrm{BF}$. At the completion of these five baseline test sessions, a series of manipulations to the hasic test schedule was instituted.

First, the stimulus duration was varied to values of 25 or 100 csec, each session preceded by a baseline session. Second, in order to assess the effect of stimulus unpredictability, animals were exposed to variations in the ITI duration: a short ITI schedule $(1.5,3.0$, and $4.5 \mathrm{sec})$ and a long ITI schedule (4.5, $6.0,7.5$, and $9.0 \mathrm{sec}$ ), each preceded by a baseline session. Equal numbers of each ITI were randomly presented during the 100 trial sessions.

Third, bursts $(0.5 \mathrm{sec})$ of distracting white noise $(105 \mathrm{~dB}$ as measured with a Dawe's sound level indicator) were interpolated at various points within the normal $5 \mathrm{sec}$ ITI: $0.5,2.5,4.5$, or $5.0 \mathrm{sec}$ after the beginning of the ITI. In the latter case, the white noise was presented simultaneously with the visual target stimulus. Within this session, no noise was presented on $20 \%$ of the trials, these trials being randomly interspersed throughout the 
Table 1. Effects of AMPA-induced lesions of the basal forebrain on regional ChAT activity in the cortex (mean $\pm \mathrm{SE}$ )

\begin{tabular}{|c|c|c|c|}
\hline \multirow[b]{2}{*}{ Region } & \multicolumn{2}{|c|}{$\mu \mathrm{mol}$ ACh formed/gm tissue $/ \mathrm{hr}$} & \multirow{2}{*}{$\begin{array}{l}\% \text { reduc- } \\
\text { tion }\end{array}$} \\
\hline & Control & Lesion & \\
\hline \multicolumn{4}{|l|}{ Experiment $1^{\prime \prime}$} \\
\hline MF & $2.659 \pm 0.11$ & $1.695 \pm 0.10$ & $36 \% * *$ \\
\hline $\mathrm{C} 1$ & $1.753 \pm 0.13$ & $1.137 \pm 0.05$ & $37 \% * *$ \\
\hline $\mathrm{C} 2$ & $2.234 \pm 0.10$ & $1.362 \pm 0.07$ & $39 \% * *$ \\
\hline ADLl & $1.196 \pm 0.11$ & $0.411 \pm 0.05$ & $66 \% * *$ \\
\hline ADL2 & $1.374 \pm 0.15$ & $0.367 \pm 0.05$ & $73 \% * *$ \\
\hline Parietal & $1.421 \pm 0.14$ & $0.412 \pm 0.03$ & $71 \% * *$ \\
\hline Hippocampus & $1.979 \pm 0.21$ & $2.175 \pm 0.14$ & NS \\
\hline \multicolumn{4}{|l|}{ Experiment $2^{b}$} \\
\hline MF & $2.05 \pm 0.17$ & $1.04 \pm 0.06$ & $50 \% * *$ \\
\hline $\mathrm{Cl}$ & $1.81 \pm 0.16$ & $1.00 \pm 0.16$ & $47 \% * *$ \\
\hline $\mathrm{C} 2$ & $1.76 \pm 0.17$ & $1.00 \pm 0.09$ & $43 \% * *$ \\
\hline ADLl & $1.76 \pm 0.09$ & $0.46 \pm 0.05$ & $74 \% * *$ \\
\hline ADL2 & $1.97 \pm 0.14$ & $0.59 \pm 0.05$ & $70 \% * *$ \\
\hline Hippocampus & $2.31 \pm 0.09$ & $2.34 \pm 0.24$ & NS \\
\hline
\end{tabular}

Control, $n=7$; lesion, $n=15$.

"Control, $n=6$; lesion, $n=6$.

${ }^{* *} p<0.001$, Student's $t$ test.

100 trial sessions. This final behavioral challenge occurred approximately 16 weeks following surgery.

Finally, in order to assess cholinergic specificity of the effects, reversal of the behavioral deficit was attempted by systemic physostigmine treatment in those animals continuing to demonstrate a deficit in performance on the baseline schedule of the task (see below). Physostigmine salycilate (dose calculated as free base) was dissolved in $0.9 \%$ saline and administered intraperitoneally $20 \mathrm{~min}$ prior to the start of the test session. Animals received a vehicle injection and three doses of physostigmine $(0.025,0.05$, and $0.1 \mathrm{mg} / \mathrm{kg})$ according to a Latin Square design. A rest day followed by a baseline day separated each drug test day. This final challenge was completcd approximatcly 20 wecks postsurgery.

\section{Results}

\section{Neurochemical results}

As shown in Table 1, infusions of AMPA into the basal forebrain resulted in significant reductions in ChAT activity in all cortical regions assayed, with the exception of the hippocampus where $\mathrm{ACh}$ levels were unaffected by the lesion. The reduction in cortical ChAT activity ranged from approximately $38 \%$ in medial areas of cortex (MF, C1, and $\mathrm{C} 2$ ) to $70 \%$ in more lateral cortical areas (ADL1, ADL2, and parietal).

\section{Histology}

Examination of cresyl violet-stained material confirmed the loss of magnocellular neurons from the region of the $n b M$ in those brains that had received AMPA infusions into the BF. However, in marked contrast to earlier histological analysis of AMPAinduced lesions of the BF (Page et al., 1991; Page and Everitt, 1993), in the $\mathrm{BF} / 1$ lesion group (see Behavioral results, immediately below) there was clear damage to the dorsal and ventral globus pallidus (see Fig. 1), including the presence of extracellular deposits that previously had only been seen following infusions of ibotenic acid into the same site (Everitt et al., 1987). In the $\mathrm{BF} / 2$ lesion group the dorsal pallidal damage was much less extensive and was predominantly restricted to the nbM, ventral pallidum, and adjacent BF. In both lesion groups there was also appreciable damage to the reticular nucleus of the thalamus and the presence of extracellular deposits in reticular and ventral thalamic nuclei. Similar pathology has previously been noted in animals with lesions of the BF that survived long postsurgical periods such as the time course of the present study (Fine et al., 1985; Wozniak et al., 1989). Analysis of these deposits (Wozniak et al., 1989) has revealed that they represent a form of calcification that closcly represents the calcospherites frequently found in patients with Alzheimer's disease (Mann, 1988) and may be a characteristic of the accumulation of calcium deposits in response to degeneration of the nbM neurons. Furthermore, there was marked gliosis in the region of the infusion that was again unusual when taken within the context of our earlier studies (Page et al., 1991; Page and Everitt, 1993).

\section{Behavioral results}

Three weeks postlesion. As shown in Figure 2A, AMPA-induced lesions of the $\mathrm{BF}$ produced a significant reduction in choice accuracy $[F(1,20)=21.98, p<0.001]$ that remained constant over the 5 d postoperative testing period, yet was above chance $(20 \%)$ levels of task performance. This reduction in accuracy of performance was accompanied by a significant lengthening of the latency to respond correctly to the visual stimulus $[F(1,20)$ $=13.86, p<0.001$; see Fig. $2 B]$. The lesion group did not show an increase in errors of omission $[F(1,20)=0.17, p>0.05$ (controls, $\mathrm{CON}=18.83 ; \mathrm{BF}=20.26$ )], or a lengthening of the latency to collect earned food reward $[F(1,20)=2.44, p>0.05$ $(\mathrm{CON}=1.90 ; \mathrm{BF}=2.44 \mathrm{sec})]$.

Perseverative responses were, however, significantly increased in the $\mathrm{BF}$ lesion group, an effect that was also dependent upon the day of testing $[F(4,78)-3.04, p<0.03]$. As shown in Figure $2 C$, the high level of perseverative responding by animals of the $\mathrm{BF}$ lesion group on the first test day continued to increase over the first three test sessions, after which time a plateau in responding was obtained. In contrast, a reduction in

Figure 1. Photomicrographs of the basal forebrain in vehicle-infused control $(A, B)$ and AMPA-infused $(C-F)$ animals. The material in $E$ and $F$ is typical of animals in group BF/1 (AMPA batch 06175), while that in $C$ and $D$ is typical of animals from experiment 2 (AMPA batch 08403). $A$ and $B$, Aggregations of large, hyperchromatic neurons of the nbM can be seen in the ventromedial pallidum and within the so-called substantia innominata (arrows). Similar large neurons of the diagonal band are visible more ventrally. The area of the nbM is shown at higher magnification in $B$, where hyperchromatic, magnocellular neurons are clearly visible (arrowhead). $C$ and $D$, The same areas at the same magnifications as in $A$ and $B$. No magnocellular neurons can be seen in the region of the nbM (arrow), yet neurons are visible elsewhere in the dorsal pallidum (around open arrow dorsally). Gliosis associated with the infusions can also be seen (arrowhead). The areas of marked magnocellular neuronal loss and associated gliosis are seen clearly in $D . E$ and $F$, Marked neuronal loss throughout the pallidum and the presence of hyperchromatic extracellular deposits in the region of the nbM (arrow in $E$, double arrow in $F$ ). There is also a more intense gliosis. Stars indicate the same areas in $A$ and $B$, $C$ and $D, E$ and $F ; m=$ medial; $v=$ ventral; $d g p$, dorsal globus pallidus. Scale bars, $100 \mu \mathrm{m}$. 

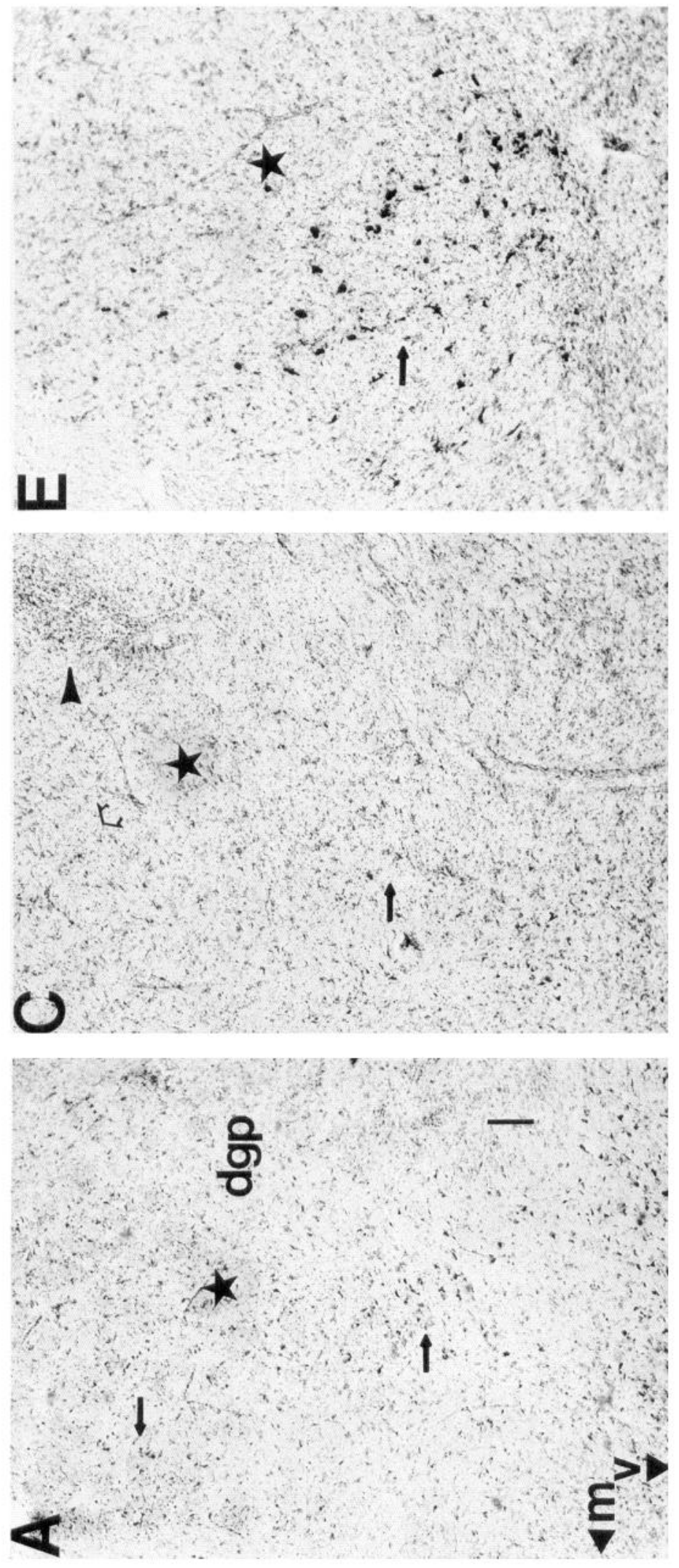

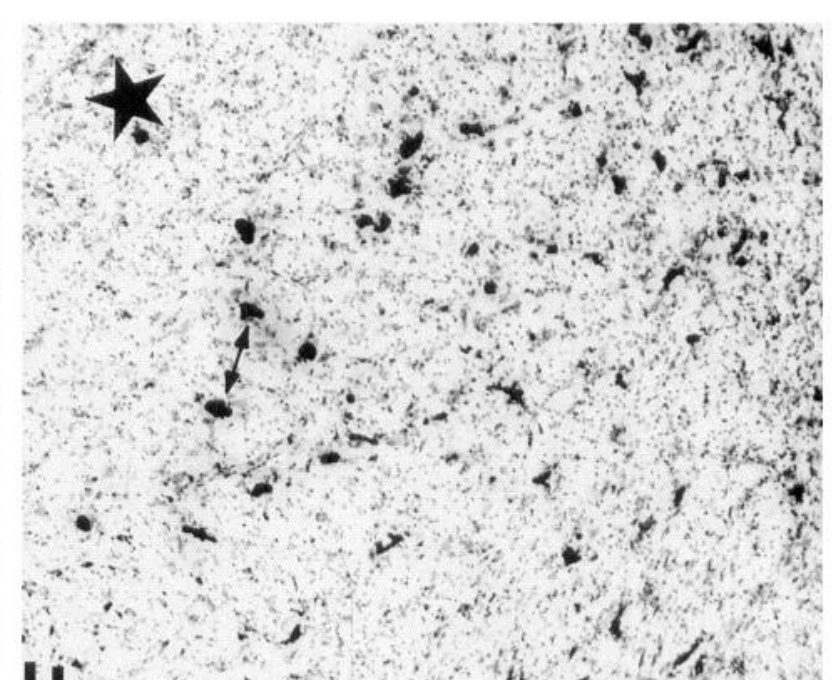

LI

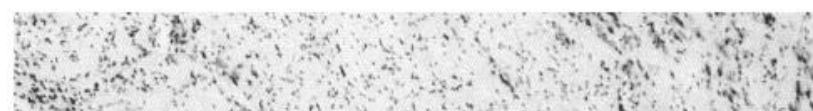

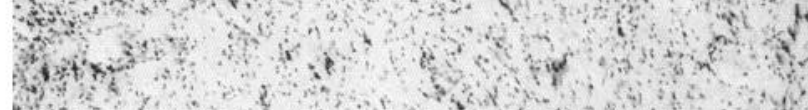

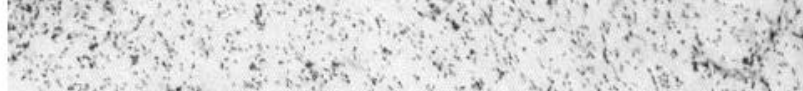

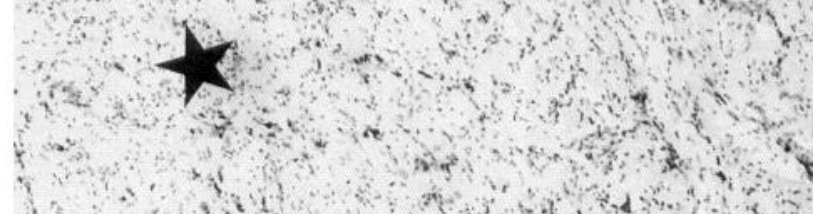

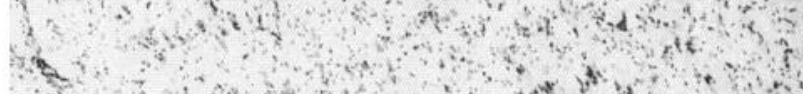

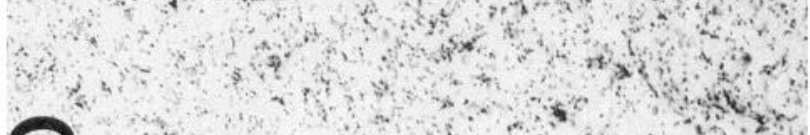

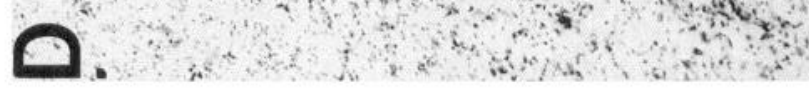

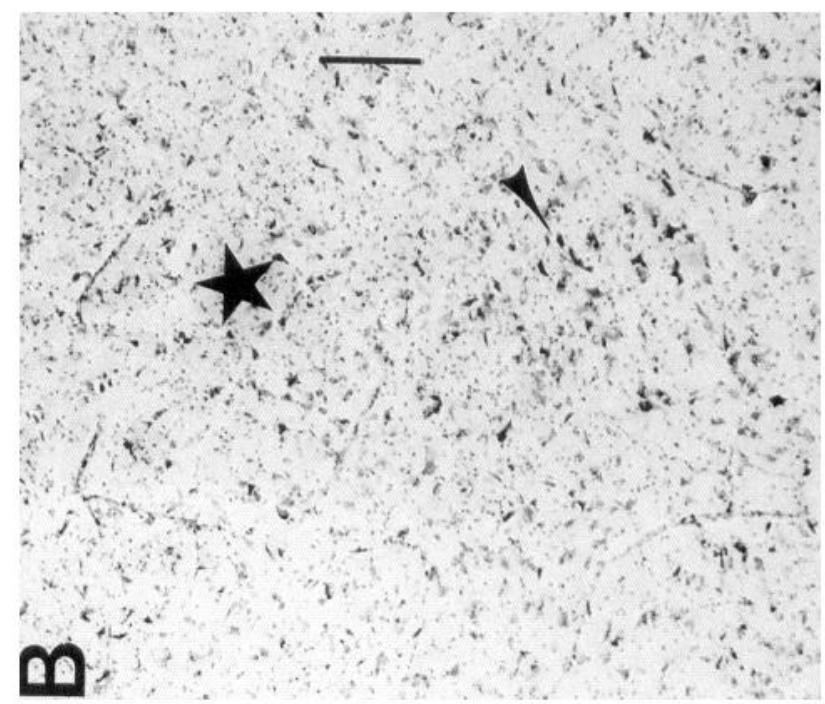



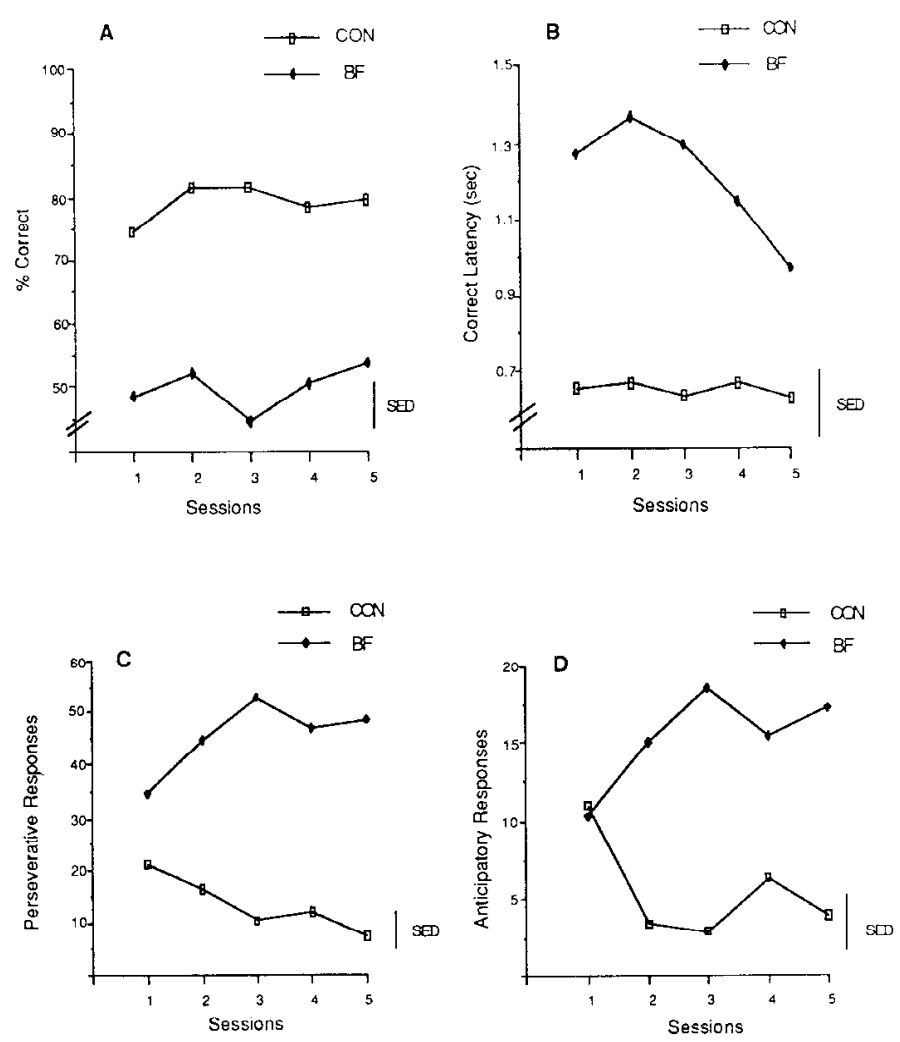

Figure 2. Performance of sham controls $(C O N)$ and AMPA-lesioned animals $(B F)$ on the baseline schedule of the task 3 weeks postlesion surgery: choice accuracy $(A)$, correct response latency $(B)$, perseverative responding $(C)$, and anticipatory responding $(D)$.

perseverative responding was observed across sessions for shamoperated controls. A significant lesion $\times$ session interaction was also found for anticipatory responding $[F(4,78)=3.71, p<$ 0.011 . Newman-Keuls post hoc comparisons revealed that anticipatory responding by animals of the BF lesion group was not significantly different from controls on the first day of testing. However, on subsequent days, the lesion group showed a significant increase in anticipatory responding with a corresponding decline in responding by control animals after the first test session (see Fig. 2D).
Fourteen weeks postlesion. Similar to the deficits observed 3 weeks postoperatively, a significant reduction in choice accuracy $[F(1,20)=5.10, p<0.05]$ and lengthening of correct response latencies $[F(1,20)=4.48, p<0.05]$ were still apparent several months following the lesion. As observed immediately following lesion surgery, there was no significant effect of the AMPAinduced basal forebrain lesion on the latency to collect food reward $[F(1,20)=0.69, p>0.05]$ or on errors of omission $[F(1,20)=2.02, p>0.05]$. The significant increase in perseverative responding in the $\mathrm{BF}$ lesion group observed 3 weeks postlesion surgery was still apparent $[F(1,20)=12.68, p<0.01]$ and remained constant across the five test sessions (means: CON $=18.4 ; \mathrm{BF}=44.8)$. The significant increase in anticipatory responding observed initially was no longer apparent $[F(1,20)$ $=1.96, p>0.05]$.

However, upon closer examination of the baseline scores obtained at this time, the BF lesion animals could clearly be divided into two groups on the basis of their performance of the task. One group of animals $(\mathrm{BF} / 1 ; n=7)$ continued to show substantial behavioral deficits on the task, while a second group $(\mathrm{BF} / 2 ; n=8)$ performed the task at a similar level to sham control animals (Fig. 3). Indeed, ANOVA revealed a significant effect of BF group on three behavioral measures: choice accuracy $[F(2,19)=16.75, p<0.001]$, correct latency $[F(2,19)=16.96$, $p<0.001$; see Fig. 3], and perseverative responding $[F(2,19)=$ $7.85, p<0.01]$. Newman-Keuls post hoc comparisons confirmed that the $\mathrm{BF} / 1$ group showed a significant reduction in choice accuracy and a significant increase in latency to respond correctly to the visual stimulus compared to animals of the sham control group and those of the $\mathrm{BF} / 2$ lesion group $(p<0.01)$. The BF/ 2 group did not differ significantly from sham controls on either of these measures. In contrast, perseverative responding was significantly increased $(p<0.05)$ in both $\mathrm{BF} / 1$ and $\mathrm{BF} / 2$ lesion groups compared to sham controls (mean perseverative scores across five sessions: $\mathrm{CON}=18.4 ; \mathrm{BF} / 1=39.1 ; \mathrm{BF} / 2=$ 51.3).

The differential performance of these two groups of lesion animals on the task was also reflected in the degree of nonspecific pallidal damage observed in these animals (see details above). Animals of the BF $/ 1$ group showed large areas of gliosis and extensive cell death within the dorsal and ventral pallidum compared to the $\mathrm{BF} / 2$ group, which showed more restricted ventral
Figure 3. Performance of sham controls $(C O N)$ and $\mathrm{BF} / 1$ and $\mathrm{BF} / 2$ lesion groups on the baseline schedule of the task 14 weeks following lesion surgery.
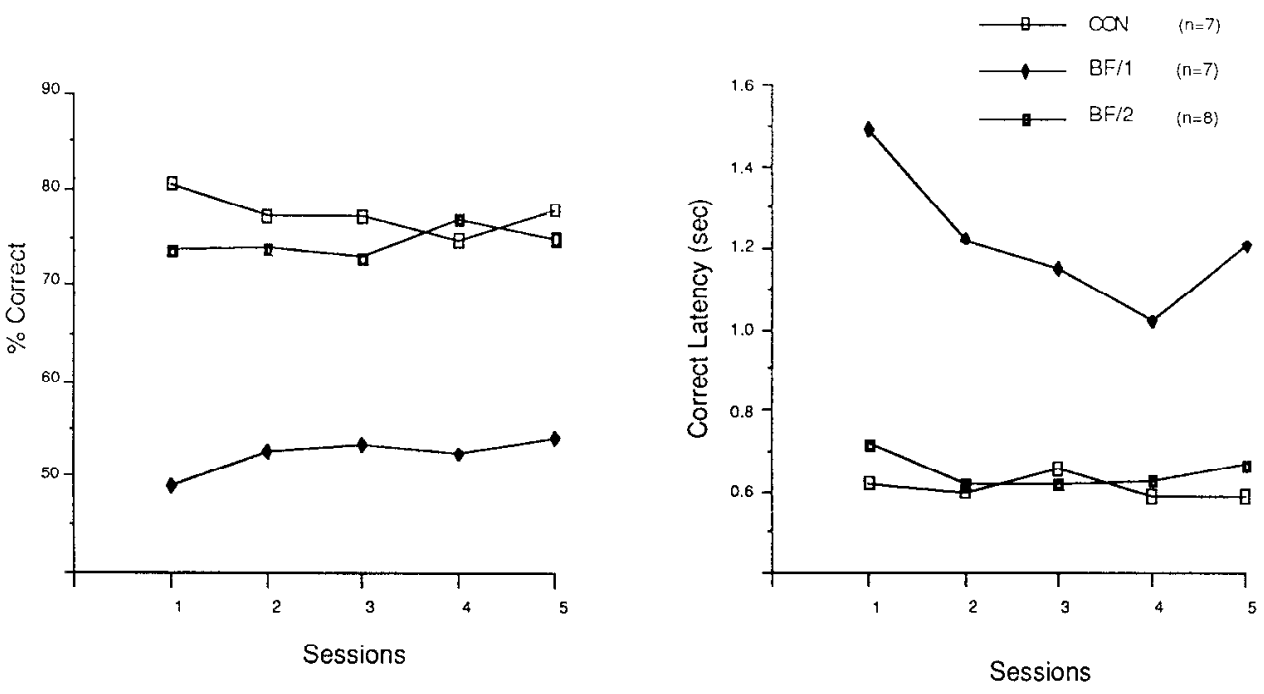
pallidal damage (see Fig. 1). Allocation of the lesion animals to two subgroups was therefore possible not only on the basis of task performance several months following the lesion surgery, but also according to subsequent histological analysis of these animals. Furthermore, these two groups of animals could be distinguished by degree of reduction of ChAT activity within anterior dorsolateral (ADL1) cortex, a significantly greater reduction in ChAT activity being observed in this region for animals of the BF/1 compared to the BF/2 lesion group (Table 2).

Following determination of baseline performance, all animals were exposed to subsequent behavioral challenges, including unpredictable presentations of short (1.5-4.5 sec) and long (4.5$9.0 \mathrm{sec})$ ITIs. However, those animals continuing to demonstrate significant behavioral deficits on the baseline schedule several months following the lesion (BF/1) and their sham controls were subsequently exposed to a lengthening of the stimulus duration $(1.0 \mathrm{sec})$ and to peripheral administration of the anti-cholinesterase physostigmine in attempts to improve task performance. In contrast, in an attempt to reinstate the performance deficits observed by animals of the $B F / 2$ lesion group immediately postoperatively, the duration of the visual stimulus was reduced $(0.25 \mathrm{sec})$ and distracting bursts of white noise were interpolated during the $5 \mathrm{sec}$ ITI.

\section{Behavioral challenges (15 weeks postlesion)}

Effect of varying the stimulus duration. Figure $4 A$ clearly shows that increasing the stimulus duration to $1.0 \mathrm{sec}$ resulted in a significant improvement in the accuracy of performance of the $\mathrm{BF} / 1$ lesion group $[F(1,12)=5.41, p<0.05]$. Post hoc comparisons confirmed this improvement in performance $(p<0.01)$, although choice accuracy for this lesion group continued to be significantly lower than for sham controls. The latency to respond correctly to the visual stimulus remained significantly lengthened in the $\mathrm{BF} / 1$ group $[F(1,12)=15.37, p<0.01]$. Although it was not significantly reduced by increasing the stimulus duration $[F(1,12)=1.95, p>0.05]$, there was a trend for this to occur $(0.50 \mathrm{sec}: \mathrm{CON}=0.59, \mathrm{BF} / 1=1.29 \mathrm{sec} ; 1.0 \mathrm{sec}$ : $\mathrm{CON}=0.65, \mathrm{BF} / 1=1.10 \mathrm{sec})$.
Table 2. Effect of AMPA lesions of the basal forebrain on reduction in ChAT activity in cortical regions for $B F / 1$ and $B F / 2$ animals (experiment 1) and AMPA animals (experiment 2)

\begin{tabular}{llll} 
& \multicolumn{2}{l}{$\%$ reduction } & \\
\cline { 2 - 4 } Region & $\mathrm{BF} / 1(n=7)$ & $\mathrm{BF} / 2(n=8)$ & $\begin{array}{l}\text { AMPA } \\
(n=6)\end{array}$ \\
\hline MF & $40 \%$ & $33 \%$ & $50 \% *$ \\
C1 & $39 \%$ & $32 \%$ & $47 \%$ \\
C2 & $42 \%$ & $36 \%$ & $43 \%$ \\
ADL1 & $75 \% \dagger$ & $57 \%$ & $74 \% *$ \\
ADL2 & $74 \%$ & $73 \%$ & $70 \%$ \\
Parietal & $71 \%$ & $71 \%$ & - \\
Hippocampus & Nil & Nil & Nil \\
\hline
\end{tabular}

* Significant reduction in ChAT compared to $\mathrm{BF} / 2(p<0.05)$.

+ Significant reduction in ChAT compared to $\mathrm{BF} / 2(p<0.05)$.

While errors of omission were significantly increased in the $\mathrm{BF} / 1$ group, independent of stimulus duration $[F(1,12)=13.81$, $p<0.01]$, they were not excessive $(\mathrm{CON}=7.50 ; \mathrm{BF} / 1=19.29)$. Once again, perseverative responding was significantly increased in the lesion group $[F(1,12)=15.85, p<0.01]$ compared to the sham controls but was unaffected by changes in the duration of the stimulus. There was no significant effect of the lesion or of this manipulation to the basic test schedule on anticipatory responding.

As shown in Figure $4 B$, reducing the stimulus duration to $0.25 \mathrm{sec}$ resulted in a marked reduction in the accuracy of performance by animals of the $\mathrm{BF} / 2$ lesion group $[F(1,13)=6.87$, $p<0.03$ ]. Newman-Keuls post hoc comparisons revealed a significant reduction in performance of both control and lesion animals when the stimulus duration was reduced, compared to performance during the baseline $(0.50 \mathrm{sec})$ stimulus condition. However, post hoc comparisons revealed that performance of the $B F / 2$ group was disrupted to a significantly greater extent by this manipulation, even though under baseline conditions their performance was equivalent to that of control animals.

Latency to respond correctly to the visual stimulus was similar

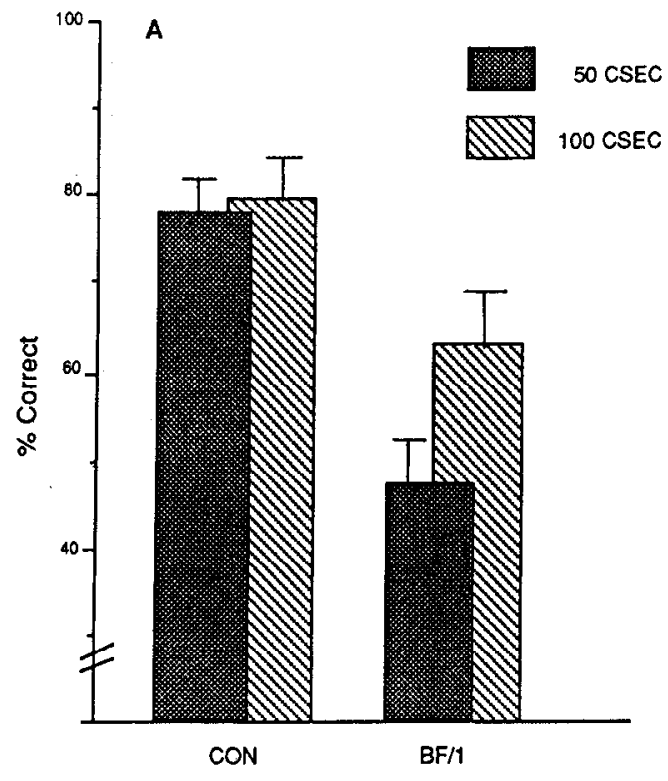

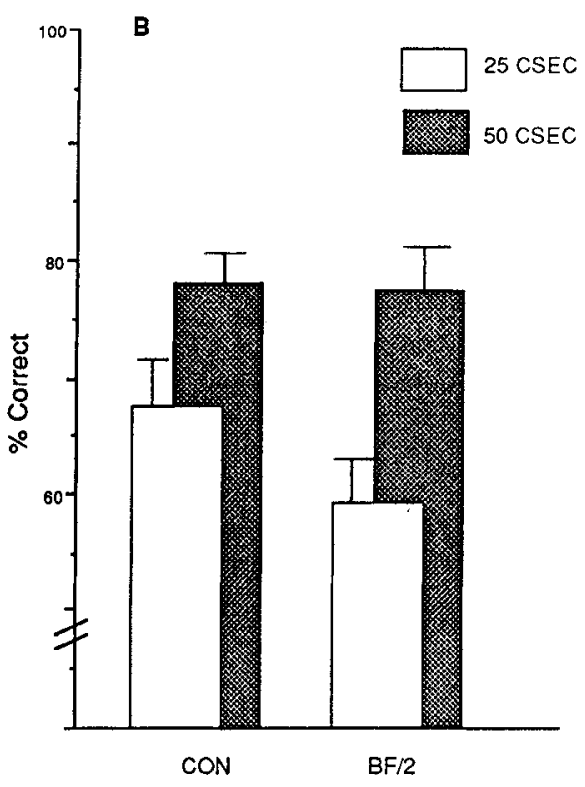

Figure 4. Effects of manipulating the duration of the visual stimulus on choice accuracy: $A$, increasing stimulus duration to $1 \mathrm{sec}$ for animals of the $\mathrm{BF} / 1$ lesion group; and $B$, reducing the stimulus duration to $0.25 \mathrm{sec}$ for animals of the $\mathrm{BF} / 2$ lesioned group. 
for both CON and BF/2 groups and was not significantly affected by variations in stimulus duration $[F(1,13)=0.42, p>0.05]$. However, latency to collect food reward was significantly affected, depending on the duration of the stimulus $[F(1,13)=$ $10.86, p<0.01$ ]. Newman-Keuls post hoc comparisons $(p<$ 0.05 ) revealed that this was predominantly due to the control group being faster to collect reward when the stimulus was shorter compared to their latencies under baseline conditions $(0.50$ sec: $\mathrm{CON}=154 ; \mathrm{BF} / 2=173 \mathrm{csec} ; 0.25 \mathrm{sec}: \mathrm{CON}=183 ; \mathrm{BF} / 2$ $=166 \mathrm{csec}$ ).

Effect of variable intertrial intervals. Unpredictable presentation of short ITIs $(1.5-4.5 \mathrm{sec})$ did not significantly disrupt the accuracy of performance of cither the $B F / 1$ or the $B F / 2$ lesion groups, these animals performing at the same level of accuracy as control animals at all ITIs tested $[F(4,38)=2.30$, $p>0.05]$. However, animals of the $\mathrm{BF} / 1$ and $\mathrm{BF} / 2$ groups took significantly longer than controls to respond correctly to the visual stimulus $(\mathrm{CON}=0.064 ; \mathrm{BF} / 1=0.89 ; \mathrm{BF} / 2=0.81 \mathrm{sec})$, an effect that was independent of the ITI length $[F(2,19)=6.98$, $p<0.01]$. There was no significant effect of this manipulation on any other behavioral measure recorded.

For animals of the $\mathrm{BF} / 1$ and $\mathrm{BF} / 2$ lesion groups, choice accuracy was not significantly affected by unpredictable presentation of long (4.5-9.0 sec) ITIs. Similarly, latency to respond correctly to the visual stimulus, latency to collect reward, and anticipatory and perseverative responding were not significantly altered by this manipulation. However, all animals showed a significant increase in perseverative $[F(3,57)=3.43, p<0.05]$ and in anticipatory $[F(3,57)=25.19, p<0.01]$ responding with increasing ITI. In addition, a significant interaction between ITI and lesion was observed for errors of omission $[F(6,57)=3.71$, $p<0.01]$. While omitted responses remained constant in the lesion animals across each of the ITIs, for the CON group a significant increase in omissions was observed at the longest ITI compared to the shorter ITIs (CON: ITI $4.5 \mathrm{sec}=1.57$; ITI 9.0 $\mathrm{sec}=6.00 ; \mathrm{BF} / 1:$ ITI $4.5 \mathrm{sec}=6.57 ;$ ITI $9.0 \mathrm{sec}=5.71 ; \mathrm{BF} / 2$; ITI $4.5 \mathrm{sec}=5.00$; ITI $9.0 \mathrm{sec}=4.38$ ).

Effect of interpolated bursts of white noise. Interpolating bursts of white noise at a level of $105 \mathrm{~dB}$ revealed a strong trend for performance to be disrupted in the $\mathrm{BF} / 2$ lesion group by this distractor, although these effects were not statistically significant: choice accuracy $[F(1,14)=3.67, p=0.07(\mathrm{CON}=78.6 \%$; $\mathrm{BF}=70.9 \%)]$ and latency to respond correctly $[F(1,14)=3.49$; $p=0.08(\mathrm{CON}=0.58 ; \mathrm{BF}=0.68 \mathrm{sec})]$. In particular, the $\mathrm{BF} / 2$ lesion group showed a clear trend to disruption of performance when the noise was interpolated simultaneously with the visual target $(\mathrm{CON}=76.9 \% ; \mathrm{BF}=65.3 \%)$. The remaining behavioral measures were not significantly affected by this manipulation.

Physostigmine treatment. Two way ANOVA revealed that for animals of the $\mathrm{BF} / 1$ lesion group, the effects of the lesion on task performance were still apparent at this time (20 weeks postlesion surgery): choice accuracy $[F(1,12)=49.85, p<0.001$ $(\mathrm{CON}=78.25 \% ; \mathrm{BF}=51.04 \%)]$, latency to respond correctly $[F(1,12)=10.68, p<0.01(\mathrm{CON}=0.63 ; \mathrm{BF}=1.00 \mathrm{sec})]$, perseverative responding $[F(1,12)=24.78, p<0.001(\mathrm{CON}=$ $14.7 ; \mathrm{BF}=52.6]$, and anticipatory responding $[F(1,12)=10.42$, $p<0.01(\mathrm{CON}=5.4 ; \mathrm{BF}=17.8)]$. Performance of $\mathrm{BF} / 1$ animals was not significantly improved by peripheral physostigmine treatment at any of the doses administered $(0.025,0.05$, and $0.1 \mathrm{mg} / \mathrm{kg}$ ). However, there was a trend for an improvement in accuracy following administration of the higher doses of physostigmine (vehicle: $\mathrm{CON}=79.14 \%, \mathrm{BF} / 1=45.43 \%$; physo- stigmine, $0.05 \mathrm{mg} / \mathrm{kg}: \mathrm{CON}=76.57 \%, \mathrm{BF} / 1=53.29 \%$; physostigmine, $0.1 \mathrm{mg} / \mathrm{kg}$ : $\mathrm{CON}=80.71 \%, \mathrm{BF} / 1=54.71 \%)$.

\section{Discussion}

Experiment 1 revealed that AMPA lesions of the BF, which produce extensive depletions of cortical ChAT activity, significantly disrupt attentional function. Impairment in performance of the baseline schedule of the task was observed 3 weeks following surgery in all lesion animals, specifically as a reduction in choice accuracy and a lengthening of the latency to respond correctly to the visual stimulus. Furthermore, these deficits were persistent in a subgroup of lesion animals (BF/1) and were still apparent 14 weeks following lesion surgery. However, it was possible to improve choice accuracy in this group by increasing the stimulus duration to $1.0 \mathrm{sec}$, thereby reducing the attentional load placed on control of the orienting response in these animals. By contrast, a second group of lesioned animals $(\mathrm{BF} / 2)$ were no longer impaired on the baseline task several months following the lesion. For these animals deficits in behavioral performance, as measured by choice accuracy, could be reinstated by reducing the duration of the visual stimulus to $0.25 \mathrm{sec}$, thus increasing the attentional load placed on the animals.

Although in the majority of the cortical regions examined comparable reductions in cortical ChAT activity were obtained for animals of both lesion subgroups, the reduction of activity within the anterior dorsolateral (ADL1) cortex was significantly greater in the BF/1 group. Therefore, one possibility is that the difference in the degree of behavioral impairment observed for the two groups of AMPA-lesioned animals is a consequence of the extent of loss of cholinergic functioning within this cortical region. However, this seems unlikely given the recent finding that quinolinic acid lesions of this area of cortex do not significantly affect baseline performance of the task (J. L. Muir, T. W. Robbins, and B. J. Everitt, unpublished observations). A second possibility is that the differential behavioral effects reflect the extent of nonspecific pallidal damage produced by the excitotoxin. Those animals with enduring behavioral deficits (BF/ 1) were subsequently found to have, in addition to extensive ChAT loss, substantial gliosis and neuronal loss in the ventral pallidum and also in the overlying ventromedial dorsal pallidum. Therefore, certain of the behavioral impairments observed in this group, for example, the persistent lengthening of the response latency to the visual stimulus and particularly the inability of physostigmine to improve task performance, may be attributable to disruption of corticostriatal outputs passing through the dorsal and ventral globus pallidus. For animals of the $B F / 2$ group, where behavioral recovery was observed several months following lesion surgery, considerably less nonspecific damage was observed and this was predominantly located within the ventral pallidal region. These findings are therefore consistent with the equivalent effects observed on task performance following ibotenate- and quisqualate-induced lesions of the basal forebrain (Robbins et al., 1989b).

The differences between the two AMPA-lesioned groups could subsequently be traced to variations in the potency of the batches of AMPA used during the course of these experiments (see Surgical procedures in Materials and Methods). The second experiment was originally designed in order to examine further the cholinergic specificity of the effects obtained in experiment 1 , attempting to induce a partial cholinergic lesion of the BF by reducing the concentration of AMPA infused into this region. Since the cortical depletions of ChAT activity were so extensive 


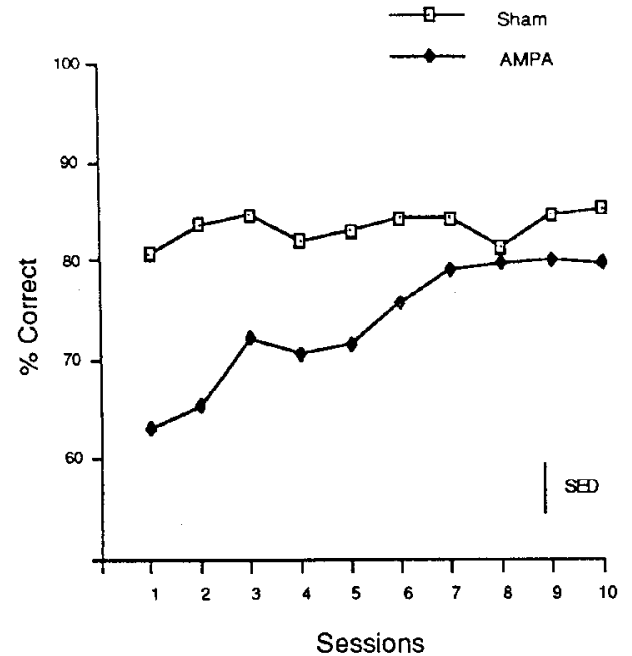

Figure 5. Performance of sham controls and AMPA-lesioned animals on the baseline schedule of the task: choice accuracy $(l e f t)$ and correct response latency (right). in experiment 1 , this may explain the difficulty in obtaining improvement of task performance using the $\mathrm{AChE}$ inhibitor physostigmine. However, following histological analysis of the lesioned animals from experiment 1 , the contribution of the second experiment became twofold: (1) enabling examination of the effects of AMPA lesions of the BF on task performance using a lesion that more closely resembled that characterized by Page et al. (1991), thus producing extensive reduction of cortical ChAT activity with minimal neuronal loss from the pallidum; and (2) allowing attempts at reversal of behavioral impairments by systemic administration of physostigmine in animals without substantial damage to the pallidal components of the corticostriatopallidal output systems.

\section{Experiment 2: Effect of AMPA Lesions of the Basal Forebrain and Systemic Physostigmine Treatment on Attentional Performance}

Results

\section{Neurochemical results}

As shown in Table 1, infusions of AMPA into the BF resulted in significant reductions in cortical ChAT activity in all cortical regions examined except for the hippocampus. Reductions in ChAT activity within the parietal cortex were unavailable due to sample loss during processing.

Further analysis was subsequently carried out in order to compare the reductions in cortical ChAT activity obtained in experiment 2 with those obtained for $B F / 1$ and $B F / 2$ animals in experiment 1. ANOVA revealed a significant effect of group, the three lesion groups showing a significant reduction in ChAT activity compared to sham controls in each region examined, with the cxception of the hippocampus. Furthermore, as shown in Table 2, within the medial frontal cortex $[F(3,33)=28.13$, $p<0.001]$ and the anterior dorsolateral (ADL1) cortex $[F(3,33)$ $=64.32, p<0.001]$, reductions in ChAT activity were significantly greater in the lesioned animals of experiment 2 than for animals of the BF $/ 2$ group $(p<0.05)$. In addition, within ADL1 only, ChAT depletion was significantly greater in the BF/ 1 group compared to $\mathrm{BF} / 2$ animals $(p<0.05)$. There were no significant differences in any region examined between ChAT depletions obtained for AMPA animals in experiment 2 and those obtained for the $\mathrm{BF} / 1$ lesion group.

\section{Histology}

Infusions of AMPA effectively destroyed the $\mathrm{Ch} 4$ cholinergic cell group but did not generally result in neuronal loss from the dorsal globus pallidus (see Fig. 1). The lesion profile of these animals was virtually identical to that reported in our earlier work (Page et al., 1991; Page and Everitt, 1993). Furthermore, the magnocellular cholinergic neurons of the vertical and horizontal limb nuclei of the diagonal band of Broca were also spared, the ventral pallidum was not damaged, and in these animals there was minimal gliosis in the region of the infusion and there were no extracellular deposits in pallidal structures or within thalamus.

\section{Behavioral results}

As shown in Figure 5, 3 weeks following lesion surgery a significant impairment in performance of the task was obscrved in lesion animals as measured by a significant reduction in choice accuracy $[F(1,10)=5.07, p<0.05]$ and a significant increase in correct response latency $[F(1,10)=4.64, p<0.05]$. This increase in response latency was also dependent upon the day of testing $[F(9,90)=4.49, p<0.001]$, animals of the lesion group responding initially with very long latencies which decreased across test sessions, returning to sham control levels.

Detailed analysis of the errors made by sham and lesion animals during the 10 baseline sessions revealed a remarkably similar distribution of errors by animals of the sham control and AMPA lesion groups. Regardless of whether the animal had the opportunity to make an incorrect response as far distant as four locations from the correct response aperture or only a maximum of two locations distant, for all animals the majority of errors were made in the aperture adjacent to the correct location (shams $=53 \% ;$ AMPA $=50 \%$ ) rather than being randomly distributed across all spatial locations. Indeed, as the distance from the correct location increased, there was a corresponding decrease in the number of errors made (shams then lesion, respectively: distance $2,25 \%, 27 \%$; distance $3,14 \%, 15 \%$; distance $4,8 \%, 8 \%$ ).

Perseverative responding was also significantly increased following the lesion $[F(1,10)=10.37, p<0.01]$ and this increased responding was maintained across the 10 baseline test sessions $(\mathrm{CON}=17.9$; AMPA $=43.4)$. Similarly, anticipatory responding was significantly increased in the lesion group after the first 


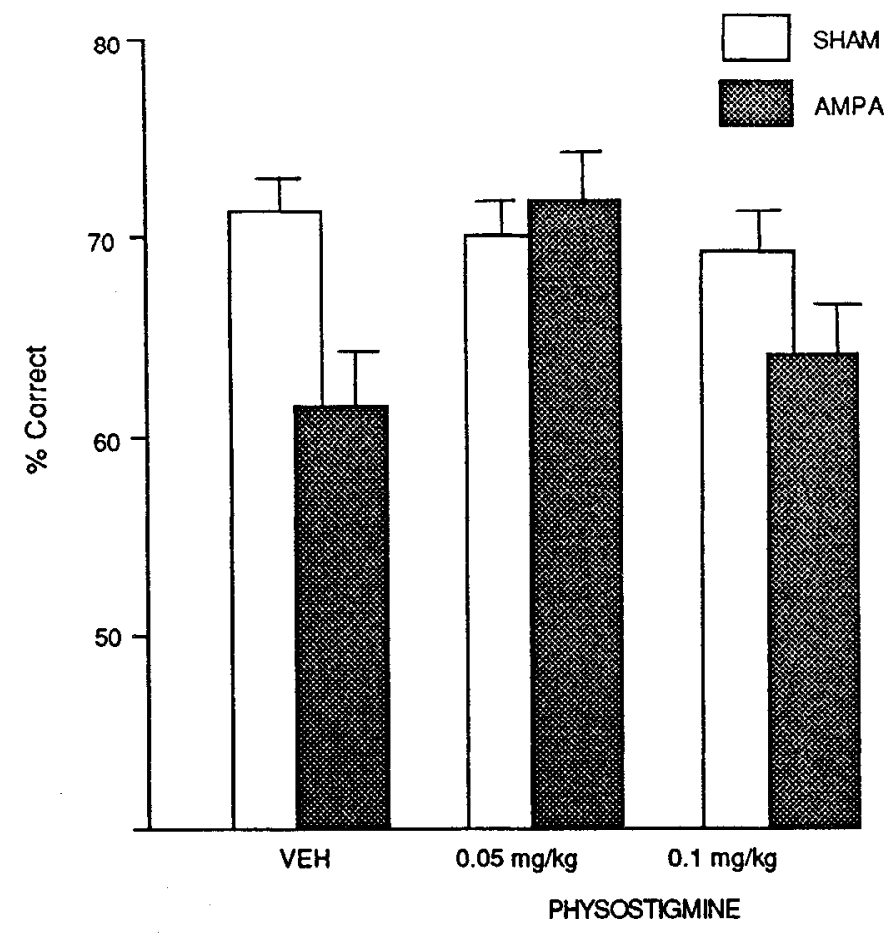

Figure 6. Effect of administration of the anti-cholinesterase physostigmine on performance of sham control and AMPA-lesioned animals using a stimulus duration of $0.25 \mathrm{sec}$.

session $[F(1,10)=24.16, p<0.001]$ and remained elevated across the remaining test sessions $(\mathrm{CON}=15.3 ; \mathrm{AMPA}=63.9)$.

However, following this $10 \mathrm{~d}$ assessment of performance, it was apparent that the lesion animals were showing behavioral recovery. All animals therefore received several additional baseline sessions in order to obtain a stable baseline upon which to initiate physostigmine treatment. At the conclusion of these additional sessions, the sham control and lesion animals did not significantly differ in their performance of the task on any behavioral measure recorded.

Consequently, the stimulus duration was reduced to $0.25 \mathrm{sec}$ for all animals to reinstate performance deficits in the lesion group and thus obtain a suitable baseline upon which to initiate physostigmine treatment in lesioned and sham control animals. This manipulation to the basic paradigm produced a significant stimulus duration $\times$ group interaction for choice accuracy $[F(1,10)=23.22, p<0.001]$. Newman-Keuls post hoc analysis revealed that a significant reduction in choice accuracy was obtained for both sham control and lesion groups when the stimulus duration was reduced to $0.25 \mathrm{sec}$. However, as observed in experiment 1 , this reduction in accuracy was significantly greater in the lesion group than in sham controls, even though performance of the two groups did not significantly differ under baseline $(0.50 \mathrm{sec})$ conditions $(0.50 \mathrm{sec}: \mathrm{CON}=83 \%$, AMPA $=82 \% ; 0.25$ sec: $\mathrm{CON}=72 \%$, AMPA $=62 \%$ ). This effect was confined to choice accuracy as there was no significant effect of reducing the stimulus duration on any other behavioral measure. AMPA animals did, however, show a transient increase in anticipatory responding which was independent of stimulus duration $(\mathrm{CON}=19.7, \mathrm{AMPA}=63.8)$.

\section{Physostigmine treatment}

As described for Experiment 1, physostigmine salycilate was dissolved in saline and administered $20 \mathrm{~min}$ prior to behavioral
Table 3. Comparison of the effects of different toxins used to lesion the BF on performance of the five-choice task 3 weeks postsurgery (0.50 sec stimulus duration) and on cholinergic depletion within frontal cortical regions

\begin{tabular}{lllll} 
& Accuracy & $\begin{array}{l}\text { Correct } \\
\text { latency }\end{array}$ & \multicolumn{2}{c}{ ChAT reductions } \\
\cline { 4 - 5 } & MF & ADLl \\
\hline BF/1 (AMPA) & $45 \%$ & 1.48 & $40 \%$ & $75 \%$ \\
BF/2 (AMPA) & $60 \%$ & 0.79 & $33 \%$ & $57 \%$ \\
Exp. 2 (AMPA) & $65 \%$ & 0.86 & $50 \%$ & $74 \%$ \\
Quisqualic acid & $72 \%$ & 0.67 & $24 \%$ & $50 \%$ \\
Ibotenic acid & $69 \%$ & 0.70 & NA & $31 \%$ \\
\hline
\end{tabular}

testing. Animals received a vehicle injection and two doses of physostigmine $(0.05$ and $0.1 \mathrm{mg} / \mathrm{kg})$ according to a Latin Square design.

Administration of physostigmine resulted in a significant dosedependent improvement in choice accuracy of performance in the lesion group $[F(2,20)-3.58, p<0.05$; Fig. 6]. Post hoc comparisons revealed that administration of $0.05 \mathrm{mg} / \mathrm{kg}$ physostigmine produced a significant increase in percentage correct responding in the lesion group compared to performance under vehicle conditions $(p<0.05)$. Indeed, performance of the lesion group under this dose of physostigmine was not significantly different from performance of sham controls. Furthermore, the improvement in task performance was specific to this particular dose, as the higher dose of physostigmine $(0.1 \mathrm{mg} / \mathrm{kg})$ did not significantly increase choice accuracy.

\section{Discussion}

Lesions of the basal forebrain induced by infusing AMPA, which produced significant depletions in cortical ChAT activity while sparing noncholinergic elements of the dorsal and ventral pallidum, resulted in a marked reduction in the accuracy of task performance and a significant lengthening of the latency to respond correctly to the visual stimulus. These behavioral deficits are qualitatively similar but greater than those obtained previously following quisqualate-induced lesions of the basal forebrain (Robbins et al., 1989a; Muir et al., 1992a). Furthermore, in the present experiment, following behavioral recovery, performance deficits could be reinstated in lesioned animals by increasing the attentional load placed on the task by reducing the duration of the visual stimulus. Importantly, the deficit in choice accuracy could be significantly improved in a dose-dependent manner by administration of the anti-cholinesterase physostigmine. This latter finding provides further support for the role of the cholinergic system in attentional function.

\section{General Discussion}

The present study investigated the effect of AMPA-induced lesions of the basal forebrain (BF) on performance of a five-choice serial reaction time task, a continuous performance test of visual attention. Previous studies (Robbins et al., 1989b; Muir et al., 1992a) have revealed markedly similar behavioral impairments in performance of this attentional task following either ibotenate- or quisqualate-induced lesions of the BF. However, the cholinergic specificity of these deficits in task performance was questionable, given the varying degree of reduction in cortical ChAT activity obtained with these two neurotoxins. The findings of the present study help to clarify this issue by revealing that AMPA, a seemingly more potent and selective neurotoxin 
for destroying cholinergic neurons, which results in even greater reductions in cortical ChAT activity than observed previously using other toxins, produces deficits in attentional performance much greater than those induced by quisqualic acid.

\section{Quantitative comparison of BF lesions}

Table 3 summarizes the effects of different toxins used to lesion the BF on performance of the five-choice task immediately postlesion and the extent of the reduction in cortical ChAT activity obtained in frontal cortical areas. Clearly, the AMPA lesions of the present study produced a more profound effect on performance of the task, as measured by choice accuracy and correct response latency, than either the quisqualate- or ibotenate-induced BF lesions of previous studies (Robbins et al., 1989b; Muir et al., 1992a). However, within the present study, three rather different $B F$ lesions were obtained depending upon the batch of AMPA supplied. As a consequence, different degrees of behavioral impairment on the task were observed.

As shown in Table 3, animals of the $\mathrm{BF} / 1$ lesion group were considerably more impaired in task performance compared to any other lesioned group. The degree of pallidal damage observed in this group was markedly similar to that obtained with ibotenic acid lesions (Robbins et al., 1989b) although the depletions of ChAT activity were substantially greater in the $\mathrm{BF} / 1$ group of the present study. This suggests that although pallidal damage combined with small reductions in cortical ChAT activity $(31 \%)$, as seen following ibotenate-induced BF lesions, impairs task performance, presumably by disrupting corticostriatal outputs passing through the pallidum, a more substantial deficit is observed when greater cortical ChAT loss is obtained, as in the case of the BF/ 1 lesion group.

When the extent of the pallidal damage was minimized (AMPA lesions of experiment 2), the behavioral impairments obtained were no longer as large as observed for the $\mathrm{BF} / 1$ lesion group, although the cortical ChAT depletions obtained for these two groups were similar. Even so, this more selective lesion of BF cholinergic neurons using AMPA produced deficits on the task that were greater than observed previously following either quisqualate- or ibotenate-induced BF lesions (Robbins et al., 1989b; Muir et al., 1992a). Furthermore, the cholinergic specificity of these deficits was supported by the attenuation of the behavioral impairments following administration of the anti-cholinesterase physostigminc, suggesting that they are due to damage to the cholinergic corticopetal projection. The pharmacological specificity of this effect is supported by the recent finding that systemic administration of amphetamine did not significantly improve task performance (Muir, Everitt, and Robbins, unpublished observations).

\section{Behavioral considerations}

The main effects of the AMPA-induced lesions of the BF were a reduction in choice accuracy and an increase in the latency to respond correctly to the visual stimulus. These impairments were accompanied by an increase in anticipatory and perseverative responding and were observed in all lesioned animals (experiments 1 and 2) immediately following surgery. Furthermore, in experiment 1 , the reduction in choice accuracy and the increase in correct response latency were still apparent in a subgroup of BF lesion animals several months following the lesion, at a time when behavioral impairments in quisqualatelesioned animals were no longer evident on the baseline schedule (Robbins et al., 1989b; Muir et al., 1992a). The increase in premature, anticipatory responding observed in the lesioned group immediately postoperatively was, however, no longer apparent and therefore appears to have been a short-lasting effect of thc lcsion. In contrast, the significant increase in perseverative responding persisted in all lesion animals. Taken together, these latter findings suggest that the mechanisms underlying premature and perseverative responding can be dissociated and may be attributable to different substrates that are probably noncholinergic and cholinergic, respectively.

Perseverative responses are generally considered a hallmark of frontal lobe dysfunction in monkeys and human, although the relationship between attentional and perseverative effects is not well understood. Shallice (1982), for example, explains attentional impairments of the type observed in the present study and perseverative deficits within the construct of a single factor related to "executive function" concerned with the allocation of attentional resources. Thus, the perseverative responding observed in the AMPA animals could also be a symptom of impaired attentional function. Alternatively, the two symptoms may be independent in psychological terms and result from damage to different regions within the prefrontal cortex. The nature of this impairment is therefore unclear at this stage.

The attentional nature of the observed deficits in experiment 1 was revealed by manipulating the duration of the visual stimulus. In those animals continuing to show a behavioral impairment on the baseline schedule of the task several months after the lesion surgery (BF/1 group), choice accuracy could be significantly improved by lengthening the duration of the visual stimulus and thus reducing the attentional load placed upon the animals, possibly by improving the precision of the orienting response. Conversely, the performance deficit in choice accuracy could be reinstated in those animals showing behavioral recovery $(\mathrm{BF} / 2$ group) by reducing the stimulus duration and thereby increasing the attentional load placed on these animals. Thus, by either decreasing or increasing the attentional load placed upon individual animals, performance of the task was improved or disrupted accordingly.

This effect was specific to choice accuracy. The lengthened response latencies observed in the $\mathrm{BF} / 1$ group of AMPA-lesioned animals were not significantly reduced by lengthening the stimulus duration, although there was a trend for this to occur. In the absence of motivational impairment and no effect of the lesion on general activity levels, the increase in correct response latencies in this subgroup of lesioned animals may be related to the decisional process involved in selection of a correct response following stimulus presentation. Therefore, although these animals showed a marked improvement in performance as measured by choice accuracy $(20 \%)$, they still required a longer period of time in which to decide upon the correct response required.

Significant changes in performance were also observed in all lesioned animals of experiment 1 when the rate of presentation of the stimuli was altered by reducing the ITI. An increase in the latency to respond correctly to the visual stimulus was observed in all animals at all shortened ITIs, although discriminative accuracy was preserved. It is unlikely that this lengthening of response latency simply reflects an impairment in motor ability, specifically in turning around to monitor the apertures for the visual target. Evidence for this suggestion is provided by the finding that a similar impairment was observed at all ITIs, including the $4.5 \mathrm{sec}$ ITI, which is only slightly shorter than the usual fixed 5 sec ITI used on the baseline schedule. Rather, 
increasing the rate of presentation of the visual stimuli by reducing the ITI, together with unpredictable presentation of the target stimuli, can be viewed as placing an additional load on attentional processing. It seems unlikely, however, that it is the temporal uncertainty of the stimuli that is the important factor for producing this deficit, given that when the ITIs were longer but still unpredictable, there was no significant effect of the lesion on response latencies. Instead, rate of stimulus presentation appears to be critical in disrupting response latencies. In terms of the Schneider and Shiffrin (1977) model of attention, this effect may be considered within the context of automatic and controlled (or effortful) processing. Automatic information processing is primarily defincd as involuntary in nature and relatively effortless, while control processing is effortful, slow, and capacity limited. If the lesion was selectively impairing effortful processing, this would explain the lengthening of response latency once the rate of stimulus presentation was increased.

Importantly, when the concentration of AMPA was reduced and a more selective cholinergic lesion was achieved (experiment 2), a similar pattern of behavioral impaiment was observed postlesion, as observed in experiment 1 . Once again, AMPA-lesioned animals showed a reduction in choice accuracy and a lengthening of response latency, accompanied by an increase in perseverative and anticipatory responding. Furthermore, as observed in the first experiment, an impairment in choice accuracy could be reinstated in lesioned animals that showed behavioral recovery by reducing the duration of the visual stimulus. It would appear that the deficit in choice accuracy, which could be improved or reinstated by varying the duration of the visual stimulus, may be attributable to disruption of the BF-cortical cholinergic projection. Support for this claim comes from the reversal of this deficit in choice accuracy by coadministration of the anti-cholinesterase physostigmine (experiment 2). In the absence of a selective cholinergic toxin, strategies such as the use of specific cholinergic agonists to attempt reversal of behavioral impairments are essential in order to infer cholinergic specificity of the observed deficits. Taken together with the results of previous work (Muir et al., 1992a) that showed amelioration of behavioral impairments on this task following transplantation of cholinergic-rich fetal tissue into the denervated neocortex, the present findings suggest that the $\mathrm{BF}$-cortical cholinergic projection is particularly involved in visual attentional function.

However, the brevity of the visual stimulus requires the animals to respond often in the absence of the stimulus, the task thus having some affinity to the delayed response task often used to assess frontal lobe dysfunction. Even so, the period of time between termination of the visual stimulus and the animal responding was usually very short, suggesting instead that the animals are in the process of making their response, that is, had oriented in the direction of the stimulus, before the stimulus had disappeared. Furthermore, Dunnett et al. (1989) have clearly shown that animals with quisqualic acid lesions of the BF do not show a delay-dependent deficit in the delayed matching-toposition task but instead show impairments in task performance even at zero delay, suggesting a disruption in attentional rather than mnemonic function. Indeed, it is now clear from a variety of studies that the nbM-cortical cholinergic neurons appear not to be essential for learning or memory per se (Dunnett et al., 1987; Etherington et al., 1987; Robbins et al., 1989a).

Detailed analysis of the location of the errors made by animals on the task revealed that approximately $50 \%$ of the errors were made by both sham and lesioned animals in the aperture adjacent to the correct location. Therefore, rather than producing a different pattern of errors compared to sham controls, the lesion simply increased the number of errors made in the vicinity of the correct location. This finding would seem to suggest that the lesion, in decreasing the attentional functioning of the animal, is reducing the accuracy with which the animal is able to locate the visual stimulus rather than disrupting performance by producing random responding across the five spatial locations. It is indeed possible that the lesion may be disrupting the spatial allocation of attention across the five spatial locations and so impair divided attention.

\section{Neural considerations}

The results of the present study strongly indicate that the BFcortical cholinergic system is important for effective attentional function. However, the cholinergic specificity of these behavioral effects cannot be assumed on the basis of AMPA-induced lesions of the BF alone. While AMPA is considerably more selective and more potent as a cholinergic toxin than either quisqualate or ibotenate, it is not a specific cholinergic neurotoxin (Page and Everitt, 1993). Quite inadvertently, during the course of these experiments we have produced lesions of the $\mathrm{BF}$, using different batches of AMPA, that resulted in large reductions in cortical ChAT activity but with varying degrees of damage to dorsal and ventral globus pallidus.

Detailed histological analysis of the lesions from experiment 1 revealed that animals of the $B F / 1$ and $B F / 2$ groups showed varying degrees of damage to dorsal and ventral globus pallidal neurons, among which the magnocellular cholinergic neurons are dispersed. In particular, animals of the $\mathrm{BF} / 1$ group were found to have considerable damage to both dorsal and ventral pallidum, while less damage was observed for animals of the $\mathrm{BF} / 2$ lesion group, being predominantly restricted to the ventral pallidum. As these pallidal neurons are important components of dorsal and ventral striatal outflow systcms (Alhcid and Hcimer, 1988), it is possible that at least some of the impairments observed in these animals were due to disruption of corticostriatal outputs that terminate on the dorsal and ventral globus pallidus neurons. It is important to note, however, that 6-hydroxydopamine lesions of the ventral striatum have previously failed to produce deficits in the accuracy of task performance and only transiently affect correct response latency on the baseline schedule (Cole and Robbins, 1989). In contrast, analysis of the lesions produced in experiment 2 revealed minimal damage to pallidal neurons.

In addition, detailed analysis of reductions in cortical ChAT activity across lesioned groups in the two experiments revealed that within the medial frontal and anterior dorsolateral (ADL1) cortices, significantly greater reductions in cortical ChAT activity were observed in the AMPA-lesioned animals of experiment 2 than for the BF/2-lesioned group of experiment 1 . However, as shown in Table 3, very similar behavioral impairments were observed in these two groups of lesioned animals. One possibility is that cholinergic functioning within these areas of frontal cortex may not be important for efficient performance of the task. However, the results of a recent study, which investigated the effect of quinolinic acid lesions of various cortical areas that are cholinergically denervated by $\mathrm{BF}$ lesions, suggest that the medial frontal cortex is indeed crucial for task performance (Muir, Robbins, and Everitt, unpublished observations). Lesions to this area of cortex produced behavioral impairments 
remarkably similar to those observed following AMPA lesions of the BF.

Alternatively, it is possible that very similar behavioral impairments are observed in the BF/ 2 lesion group, even though frontal cortical ChAT reductions are not as extensive, as a consequence of damage to the pallidal component of the corticostriatopallidal output system. As damage in this group of lesioned animals was predominantly restricted to the ventral pallidum and given that this region of pallidum has strong connections with the medial frontal cortex, it may not be surprising that disruption of task performance could be obtained either by substantial depletions of cholinergic input to the frontal cortex or by damage to the pallidal projection zone of this corticostriatopallidal loop.

This neural analysis may therefore provide important insight, for example, into the effects of the anti-cholinesterase physostigmine on task performance. Indeed, the most important finding of the second experiment was the ability of physostigmine to improve the accuracy of performance in AMPA-lesioned animals. This result is particularly important in attempting to infer cholinergic specificity of the performance deficit, given the lack of a specific cholinergic toxin. Physostigmine treatment had also been administered to animals in experiment 1 without significant effect. Taken together, these results suggest that intact cortical function is not sufficient to allow restoration of behavioral function following treatment with cholinergic agents. The inability of physostigmine to ameliorate behavioral deficits in animals that also showed significant damage to the dorsal pallidum (experiment 1) suggests the importance of uninterrupted corticostriatal outflow passing through the dorsal globus pallidus.

\section{Neurobiological implications}

The nucleus basalis has been strongly implicated in arousal processes (Buzsaki and Gage, 1990; Richardson and DeLong, 1990), and in particular it has becn proposed that during EEG desynchronization, the nbM may mediate the cortical activation component of the tonically aroused state (Steriade, 1984). Furthermore, studies of the effect of ACh in normal sensory cortex suggest that $\mathrm{ACh}$ has a role as a neuromodulator: in its presence neuronal responsiveness to afferent stimuli is enhanced and the signal-to-noise ratio is increased. Thus, without significantly increasing background activity, $\mathrm{ACh}$ has been shown to increase responses of visual cortical neurons to optimal visual stimuli (Sillito and Kemp, 1983) and a similar enhancement of signalto-noise ratio by $\mathrm{ACh}$ has been observed in the response of cortical somatosensory neurons to tactile stimuli (Donoghue and Carroll, 1987).

Phasic neuronal responses have also been documented within the nucleus basalis (Richardson and DeLong, 1991). These phasic responses appear to be related to brief deviations from baseline firing rates in awake animals whose EEG is already desynchronized and may be related to the brief change in cortical activation following, for example, a particularly salient stimulus. It is therefore possible that these phasic responses may be involved in attentional processes. Richardson and DeLong (1991) argue that one possible mechanism for attention is that increased background discharge rates in a selected group of basalis neurons could increase $\mathrm{ACh}$ release in specific cortical regions, although there is little direct evidence to suggest that this occurs.

Although the ascending projection of the cholinergic BF is extensive, innervating vast areas of cortex, it is important to consider the functions of the terminal regions that appear to be particularly important for task performance and the consequence of improvement in information processing by $\mathrm{ACh}$ in such terminal domains. Within the context of the present study, the consequence of enhancing the signal-to-noise ratio within frontal cortex requires careful consideration, given that this area of cortex appears to be particularly important for performance of the five-choice attentional task (Muir, Robbins, and Everitt, unpublished observations). Indeed, recent evidence suggests that in human subjects, the neural systems relevant to sustained attention to sensory input, as shown by regional blood flow studies, involve prefrontal cortex and superior parietal cortex (Pardo et al., 1991). Therefore, we would argue that disruption of task performance observed in the present study following AMPA-induced lesions of the BF and the improvement in performance following administration of physostigmine are probably due to alterations in functioning within the frontal cortex. Further support for this possibility comes from our previous finding that deficits in task performance could be improved by cholinergic-rich fetal tissuc transplanted into the frontoparietal cortex of lesioned animals (Muir et al., 1992a). That this improvement in performance following cholinergic replacement strategies is a result of improvement in attentional function rather than learning or memory per se is supported by the report that in primates nucleus basalis neurons that demonstrate differential delay period responses are very rarely found (Richardson and DeLong, 1986, 1990).

The results of the present study, taken together with the findings of several earlier experimental studies (Robbins et al., 1989b; Muir et al., 1992a,b), therefore support a role for the cholinergic system in control of certain attentional processes. Quite conclusively, the most consistent impairment observed following manipulation of the BF corticopetal cholinergic system is attentional in nature. The clinical relevance of these findings is demonstrated by a recent clinical report (Sahakian et al., 1993) of an improvement in attentional rather than mnemonic function in patients suffering from Alzheimer's disease following administration of the anti-cholinesterase tetrahydroaminoacradine using a computerized form of the five-choice task used in the present study.

\section{References}

Agid Y, Ruberg M, Dubois B, Pillon B (1987) Anatomoclinical and biochemical concepts of subcortical dementia. In: Cognitive neurochemistry (Stahl SM, Iversen SD, Goodman EC, eds), pp 248-271. Oxford: Oxford UP.

Alheid GF, Heimer L (1988) New perspectives in basal forebrain organization of special relevance for neuropsychiatric disorders: the striatopallidal, amygdaloid and corticopetal components of the substantia innominata. Neuroscience 27:1-39.

Bartus RT, Dean RL, Beer B, Lippa AS (1982) The cholinergic hypothesis of geriatric memory dysfunction. Science 217:408-417.

Buzsaki G, Gage FH (1990) Role of the basal forebrain cholinergic system in cortical activation and arousal. In: Activation to acquisition: functional aspects of the forebrain cholinergic system (Richardson RT, ed). Boston: Birkhauser.

Carli M, Robbins TW, Evenden JL, Everitt BJ (1983) Effects of lesions to ascending noradrenergic neurons on performance on a 5-choice serial reaction time task in rats: implications for theories of dorsal noradrenergic bundle function based on selective attention and arousal. Behav Brain Res 9:361-380.

Cole BJ, Robbins TW (1989) Effects of 6-hydroxydopamine lesions of the nucleus accumbens on performance of a 5-choice serial reaction time task in rats: implications for theories of selective attention and arousal. Behav Brain Res 33:165-179. 
Coyle JT, Price DL, DeLong MR (1983) Alzheimer's disease: a disorder of cortical cholinergic innervation. Science 219:1184-1 190.

Donoghue JP, Carroll KL (1987) Cholinergic modulation of sensory responses in rat primary somatic sensory cortex. Brain Res 408:367371 .

Dunnett SB, Whishaw IQ, Jones GH, Bunch ST (1987) Behavioural, biochemical and histochemical effects of different neurotoxic amino acids injected into nucleus basalis magnocellularis of rats. Neuroscience 20:653-669.

Dunnctt SB, Roger DC, Jones GH (1989) Effects of nucleus basalis magnocellularis lesions in rats on delayed matching and non-matching to position tasks. Eur J Neurosci 1:395-406.

Dunnett SB, Everitt BJ, Robbins TW (1991) The basal forebraincortical cholinergic system: interpreting the functional consequences of excitotoxic lesions. Trends Neurosci 14:494-501.

Etherington RE, Mittleman G, Robbins TW (1987) Comparative effects of nucleus basalis and fimbria-fornix lesions on delayed matching and alternation tests of memory. Neurosci Res Commun 1:135-143.

Everitt BJ, Robbins TW, Evenden JL, Marston HM, Jones GH, Sirkia TE (1987) The effects of excitotoxic lesions of the substantia innominata, ventral and dorsal globus pallidus on the acquisition and retention of a conditional visual discrimination: implications for cholinergic hypotheses of learning and memory. Neuroscience 22:441469.

Fibiger HC (1991) Cholinergic mechanisms in learning and memory and dementia: a review of recent evidence. Trends Neurosci 14:220 223.

Fine A, Dunnett SB, Bjorklund A, Clarke D, Iversen SD (1985) Transplantation of embryonic ventral forebrain neurons to the neocortex of rats with lesions of nucleus basalis magnocellularis-1. Biochemical and anatomical observations. Neuroscience 16:769-786.

Fonnum $F$ (1975) A rapid radiochemical method for the determination of choline acetyltransferase. J Neurochem 24:407-409.

Mann DMA (1988) The pathological association between Down syndrome and Alzheimer's disease. Mech Ageing Dev 43:99-136.

Muir JL, Dunnett SB, Robbins TW, Everitt BJ (1992a) Attentional functions of the forebrain cholinergic systems: effects of intraventricular hemicholinium, physostigmine, basal forebrain lesions and intracortical grafts on a multiple-choice serial reaction time task. Exp Brain Res 89:611-622.

Muir JL, Robbins TW, Everitt BJ (1992b) Disruptive effects of muscimol infused into the basal forebrain on conditional discrimination and visual attention: differential interactions with cholinergic mechanisms. Psychopharmacology 107:541-550.

Page KJ, Everitt BJ (1993) Transsynaptic induction of c-fos in basal forebrain, diencephalic and midbrain neurons following AMPA-induced activation of the dorsal and ventral striatum. Exp Brain Res 93:399-411.

Page KJ, Everitt BJ, Robbins TW, Marston HM, Wilkinson LS (1991) Dissociable effects on spatial maze and passive avoidance acquisition and retention following AMPA- and ibotenic acid-induced excitotoxic lesions of the basal forebrain in rats: differential dependence on cholinergic neuronal loss. Neuroscience 43:457-472.
Pardo JV, Fox PT, Raichle ME (1991) Localization of a human system for sustained attention by positron emission tomography. Nature 349: 61-64.

Richardson RT, DeLong MR (1986) Nucleus basalis of Meynert neuronal activity during a delayed response task in monkey. Brain Res 399:364.

Richardson RT, DeLong MR (1988) A reappraisal of the functions of the nucleus basalis of Meynert. Trends Neurosci 11:264-267.

Richardson RT, DeLong MR (1990) Functional implications of tonic and phasic activity changes in nucleus basalis neurones. In: Activation to acquisition: functional aspects of the forebrain cholinergic system (Richardson RT, ed). Boston: Birkhauser.

Richardson RT, DeLong MR (1991) Elcctrophysiological studics of the functions of the nucleus basalis in primates. In: The basal forebrain (Napier TC, ed), pp 233-252. New York: Plenum.

Robbins TW, Everitt BJ, Ryan CN, Marston HM, Jones GH, Page KJ (1989a) Comparative effects of quisqualic and ibotenic acid-induced lesions of the substantia innominata and globus pallidus on the acquisition of a conditional visual discrimination: differential effects on cholinergic mechanisms. Neuroscience 28:337-352.

Robbins TW, Everitt BJ, Marston HM, Wilkinson J, Jones GH, Page KJ (1989b) Comparative effects of ibotenic acid- and quisqualic acid-induced lesions of the substantia innominata on attentional function in the rat: further implications for the role of the cholinergic neurons of the nucleus basalis in cognitive processes. Behav Brain Res 35:221-240.

Sahakian BJ, Owen AM, Morant NJ, Eagger SA, Boddington S, Crayton L, Crockford HA, Crooks M, Hill K, Levy R (1993) Further analysis of the cognitive effects of tetrahydroaminoacridine (THA) in Alzheimer's disease: assessment of attentional and mnemonic function using CANTAB. Psychopharmacology 1 10:395-401.

Schneider W, Shiffrin RM (1977) Controlled and automatic information processing: 1 . Detection, search and attention. Psychol Rev $84: 1-66$.

Shallice T (1982) Specific impairments in planning. Philos Trans R Soc Lond [Biol] 298:199-209.

Sillito AM, Kemp JA (1983) Cholinergic modulation of the functional organization of the cat visual cortex. Brain Res 289:143-155.

Steriade M (1984) The excitatory-inhibitory response sequence in thalamic and neocortical cells: state regulated changes and regulatory systems. In: Dynamic aspects of ncocortical function (Edelman GM, Gall WE, Cowan WM, eds), p 107. New York: Wiley.

Wenk GL, Markowska AL, Olton DS (1989) Basal forebrain lesions and memory: alterations in neurotensin, not acetylcholine, may cause amnesia. Behav Neurosci 103:395-406.

Winer BJ (1971) Statistical principles in experimental design, 2d ed. New York: McGraw-Hill.

Wozniak DF, Stewart GR, Finger S, Olney JW (1989) Comparison of behavioural effects of nucleus basalis magnocellular lesions and somatosensory cortex ablation in the rat. Neuroscience 32:685-700.

Zilles K (1985) The cortex of the rat. Berlin: Springer. 